\title{
BASSIN VERSANT ET HYDROLOGIE DE LA RIVIERE LOT
}

\author{
par J. N. TOURENQ ${ }^{1}$, J. CAPBLANCQ ${ }^{1}$ et H. CASANOVA ${ }^{2}$
}

1) Le bassin versant du Lot $\left(11840 \mathrm{~km}^{2}\right)$ comprend trois parties aux substratums tour à tour siliceux, calcaire et alluvial, se succédant de l'amont vers l'aval.

2) La population (31 hab./ $\mathbf{k m}^{2}$ ) est en majorité rurale. Des zones incultes occupent de vastes territoires dans les parties supérieure et moyenne du bassin versant.

3) Un rythme annuel très prononcé caractérise le régime naturel du Lot. L'écoulement naturel corespond à $46 \%$ des précipitations tombées sur le bassin. Dans les parties moyenne et inférieure, une évapotranspiration importante épuise les réserves hydriques du sol pendant l'été. Divers prélèvements aggravent la sévérité des débits d'étiage.

4) Une année sur deux en moyenne, l'exploitation des grands barrages de l'amont réduit, sur une base mensuelle, de 5 à $46 \%$ les débits d'étiage réels par rapport aux débits naturels. Les débits instantanés subissent d'importantes fluctuations.

5) Un modèle des écoulements permet de déterminer la vitesse moyenne, le temps de renouvellement et le temps de propagation des ondes de débit pour la zone canalisée du Lot $(250 \mathrm{~km})$.

\section{The Drainage Basin and Hydrology of the River Lot.}

1. The drainage basin of the Lot $\left(11,840 \mathrm{~km}^{2}\right)$ is composed of three types of substratum, from source to mouth - siliceous, calcareous and alluvial respectively.

2. Most of the human population $\left(31 \mathrm{hab} . / \mathrm{km}^{2}\right)$ is rural. Large areas of the upper and middle drainage basin are uncultivated.

3. The flow regime of the Lot is characterised by a very pronounced annual rhythm. The natural flow is equivalent to $46 \%$ of the precipitation which falls in the basin. In the middle and lower parts of the basin, evapotranspiration depletes the soil of its water reserves in summer. Use of the water for various purposes increases the severity of low flows.

4. In one year in every two, on average, operation of the large barrages upstream reduces the actual monthly flow under low flow condition by $5-46 \%$. Instantaneous flows undergo marked fluctuations.

5. The average speed, the turnover time and the arrival time for flow waves for the canalised zone of the Lot $(250 \mathrm{~km})$ can be calculated from a model of discharge patterns.

1. Laboratoire d'Hydrobiologie (ERA 702), Université Paul-Sabatier, 118, route de Narbonne. 31077 Toulouse Cedex.

2. et Service de la Carte de la Végétation du C.N.R.S., 29, rue Jeanne-Marvig, 31055 Toulouse Cedex. 
Le bassin versant du Lot regroupe des ensembles très divers, avec des caractéristiques variées quant aux substrats, aux pentes et aux climats. La combinaison de ces influences naturelles a déterminé un régime d'écoulement que l'homme a fortement modifié au cours des dernières décennies en édifiant de nombreux barrages sur le cours de la rivière.

Nous présentons dans cet article une étude du bassin versant et du régime hydrologique du Lot.

\section{BASSIN VERSANT}

Le Lot prend sa source à $1295 \mathrm{~m}$ d'altitude, au Nord du Mont Lozère. Il se jette dans la Garonne à Aiguillon (alt. $22 \mathrm{~m}$ ), après un cours de $491 \mathrm{~km}$. Le bassin versant, orienté Est-Ouest, s'étend sur $11840 \mathrm{~km}^{2}$. La rivière et ses affluents drainent des milieux naturels variés, générateurs de conditions d'alimentation et d'écoulement différentes.

La région du bassin située en amont d'Entraygues (Haut Lot et Truyère), soit $46 \%$ de la superficie totale, est comprise entre 650 et 1500 mètres d'altitude (fig. 1a). Les pentes y sont fortes et l'indice d'intensité de drainage (nombre de ruisseaux d'ordre 1/superficie du bassin versant) voisin de 0,1 . En aval, la pente s'affaiblit considérablement et l'indice d'intensité de drainage est compris entre 0,07 et 0,05 .

Sol (fig. 1b). - Trois zones se succèdent de l'amont vers l'aval.

- La zone amont comprend les bassins du Haut Lot et de la Truyère ; elle se poursuit jusqu'à Capdenac. La bordure méridionale, sur la rive gauche du Lot, composée de calcaires du secondaire, forme les causses de Sauveterre, du Cantal et de Sèverac. Les sols y sont squelettiques. Le reste de cette zone amont est constitué de roches cristallophylliennes (micaschistes, granites, gneiss) et éruptives (basaltes). Sur ce substratum siliceux, relativement peu perméable, dominent des sols bruns lessivés.

- La zone moyenne, entre Capdenac et Fumel, comprend des terrains calcaires jurassiques : c'est la région des grands causses du Quercy. Les sols, peu évolués, laissent apparaître la roche mère sur de larges surfaces.

- La zone aval, entre Fumel et la Garonne, est constituée par des éléments sédimentaires du tertiaire, recouverts de sols marno-calcaires ou argilo-calcaires assez peu perméables par suite de l'importance de la fraction argilo-limoneuse. 

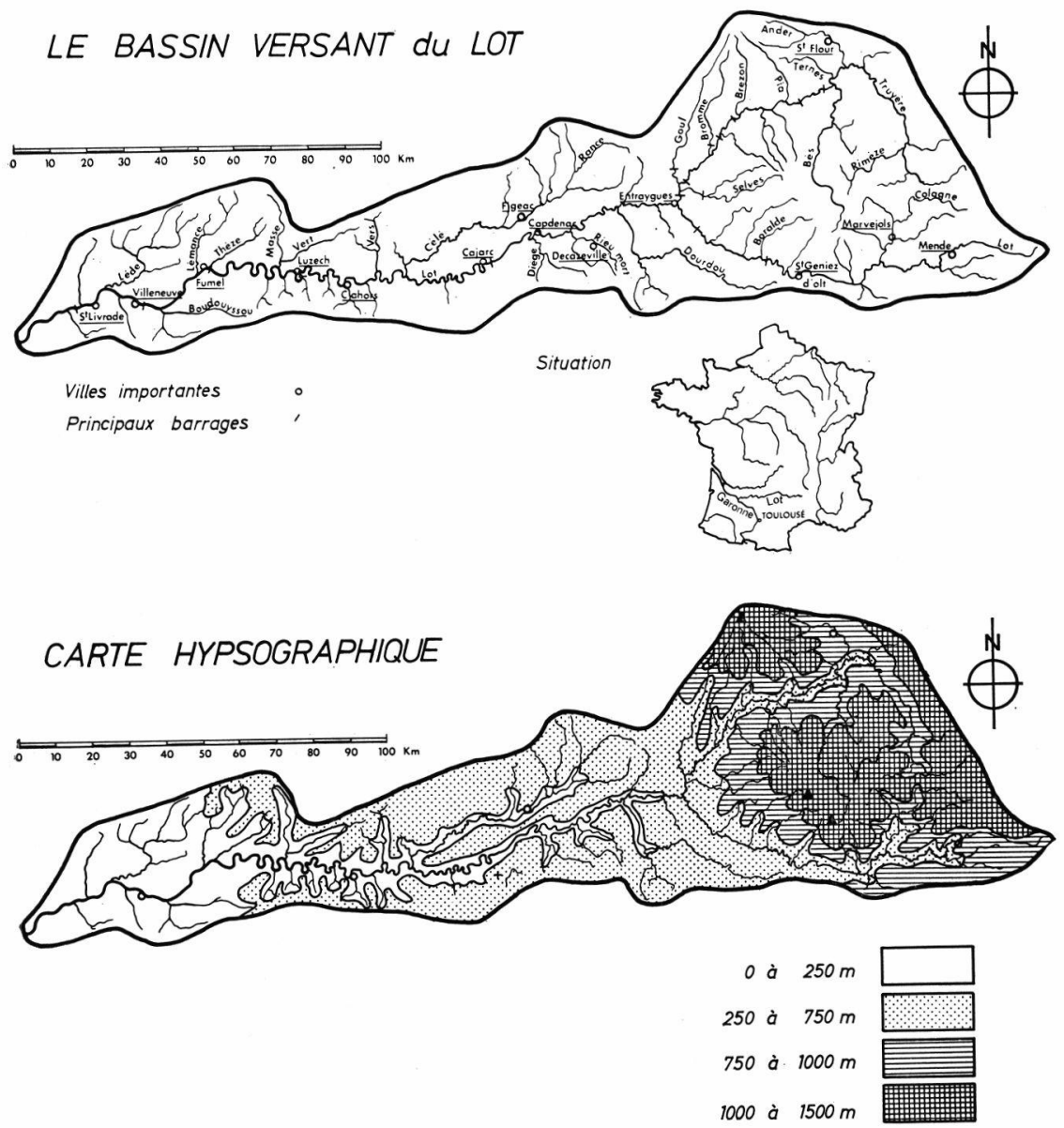

Principaux TYPES de SOL d'après

la nature de la roche mère

et leur degré de lessivage
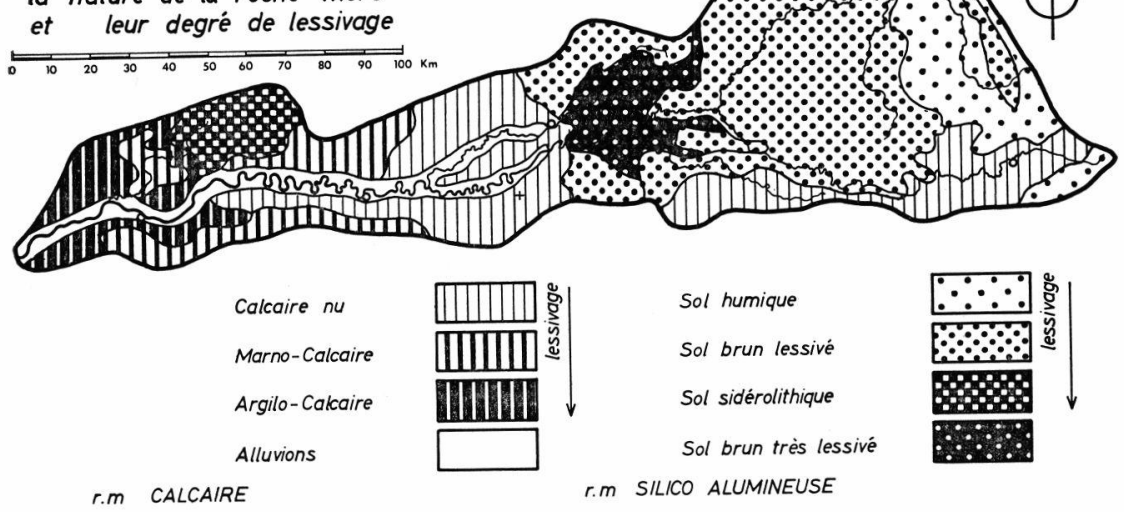

FIG. 1, 1a et 1 b. - Cartes du bassin versant du Lot. 1 : le bassin versant du Lot ; 1a: carte hypsographique; $1 \mathrm{~b}$ : principaux types de sol. 


\section{CARTE PLUVIOMÉTRIQUE}
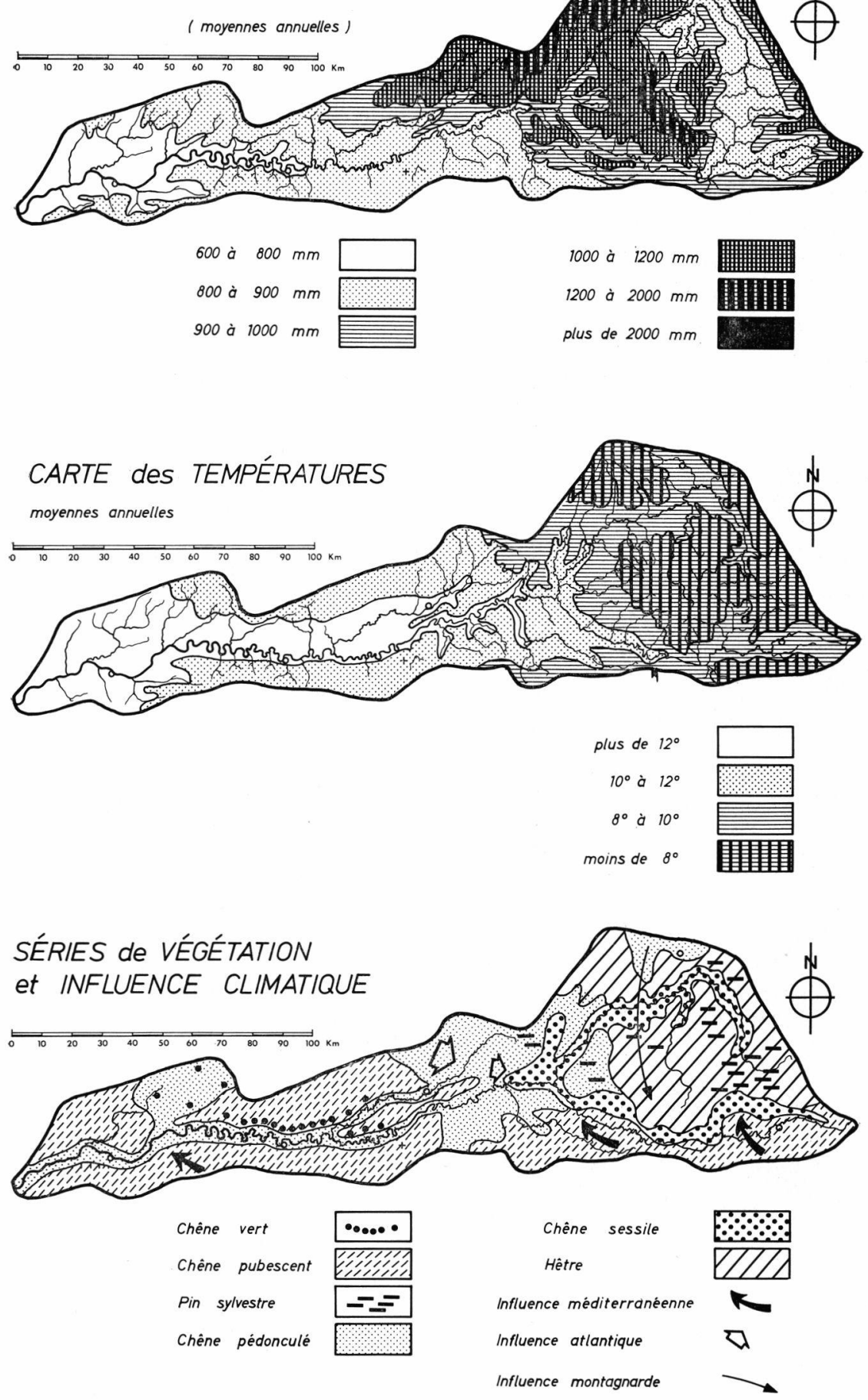

Fig. 1c à 1e. - Cartes du bassin versant du Lot. 1c: carte pluviométrique; 1d : carte des températures; le: séries de végétation et influence climatique. 

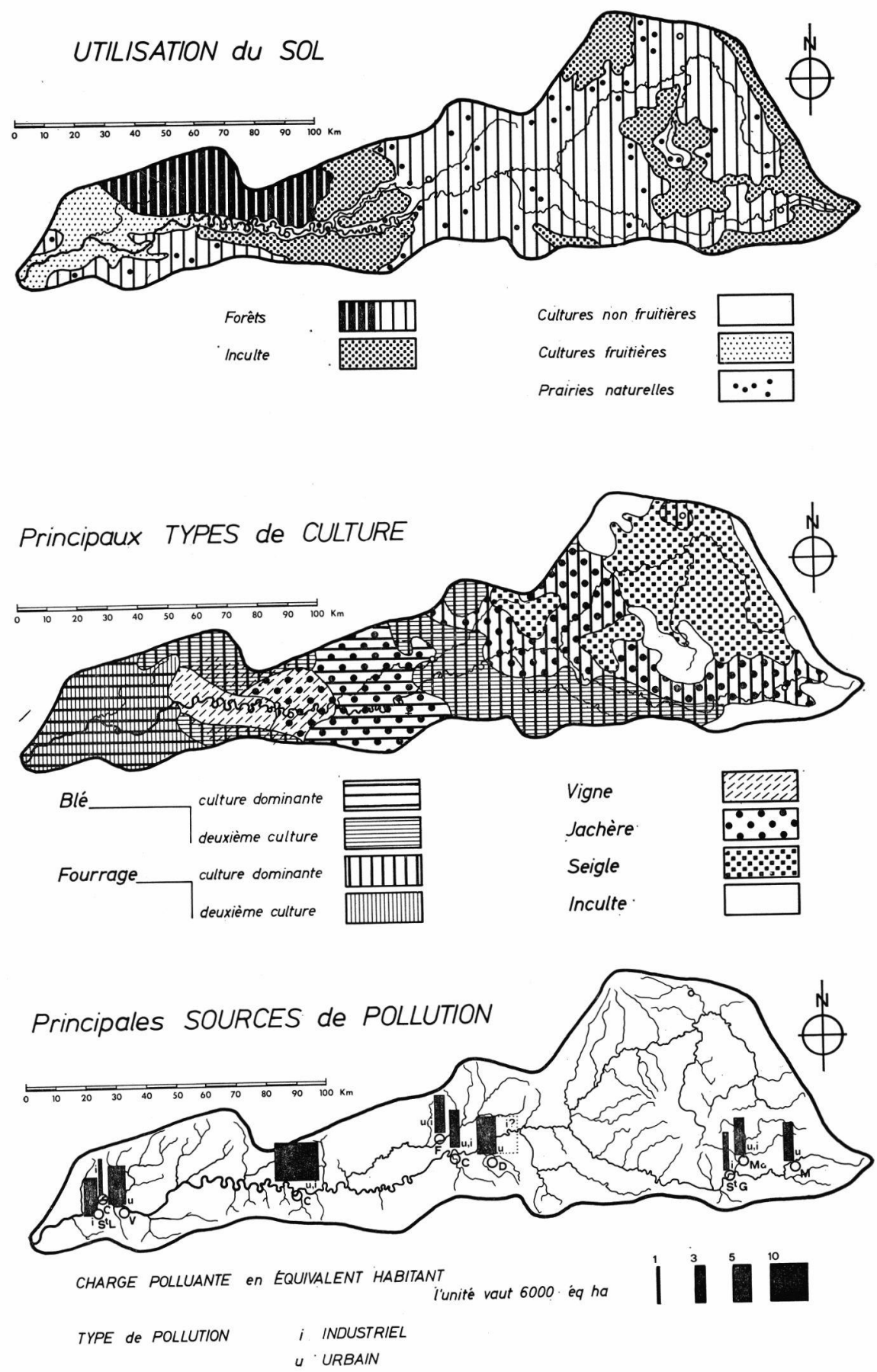

FIG. 1f à $1 \mathrm{~h}$. - Cartes du bassin versant du Lot. 1f : utilisation du sol ; $1 \mathrm{~g}$ : principaux types de culture; $1 \mathrm{~h}$ : principales sources de pollution organique. 
Climat (fig. 1c et 1d). - L'influence atlantique se manifeste par des précipitations essentiellement hivernales sur l'ensemble du bassin, l'influence méditerranéenne par des pluies d'automne sur la bordure méridionale du Haut Lot, et l'influence montagnarde par une augmentation des précipitations, notamment neigeuses, sur la partie supérieure et septentrionale du bassin.

Végétation (fig. 1c à 1g). — Les zones boisées (hêtre, chêne pédonculé) et les prairies naturelles (élevage bovin) dominent en montagne. Dans les Causses apparaît une végétation plus xérophile, avec des bois et landes des séries du chêne pubescent et du chêne vert : l'élevage ovin des plateaux fait place à une polyculture vivrière dans les dépressions argileuses. Enfin, le ruban alluvial et la basse vallée favorisent l'arboriculture et la production de céréales.

Population (fig. $1 \mathrm{~h})$. - La densité moyenne ( $\left.31 \mathrm{hab} . / \mathrm{km}^{2}\right)$ est très inférieure à la moyenne nationale. La population rurale domine netnement, mais des zones incultes, très faiblement peuplées, occupent de vastes territoires dans les parties supérieure et moyenne du bassin versant. Le secteur primaire emploie $45 \%$ des personnes actives.

Le secteur industriel, relativement peu développé, concerne :

- les industries agro-alimentaires : laits et fromages du haut bassin, fruits et légumes de la basse vallée,

- les industries extractrices et métallurgiques: bassins de Decazeville et du Fumel.

Les divers centres de la vallée sont par suite composés de villes petites ou moyennes.

\section{LES DEBITS NATURELS}

Les débits naturels représentent les apports réels des différentes régions du bassin versant. Leur calcul repose, pour chaque jour, 1) sur les données hydrométriques enregistrées aux diverses stations, 2) sur les volumes stockés ou relâchés par les grands barrages de l'amont.

Le régime naturel du Lot est caractérisé par un rythme annuel très prononcé (fig. 2) : aux précipitations d'automne et d'hiver succèdent des étés chauds et secs pendant lesquels l'évapotranspiration est intense. Une année sur 10, au confluent avec la Garonne, le débit mensuel du mois d'août tombe au-dessous de $9 \mathrm{~m}^{3} / \mathrm{s}$ pour un module annuel voisin de $150 \mathrm{~m}^{3} / \mathrm{s}$. A Cahors, le rapport des extrêmes mensuels est de 20 en moyenne pour la période de 1962 à 1975 ; il peut dépasser 40 . On observe enfin, d'une année à l'autre, des variations de modules de l'ordre de 1 à 7 . 


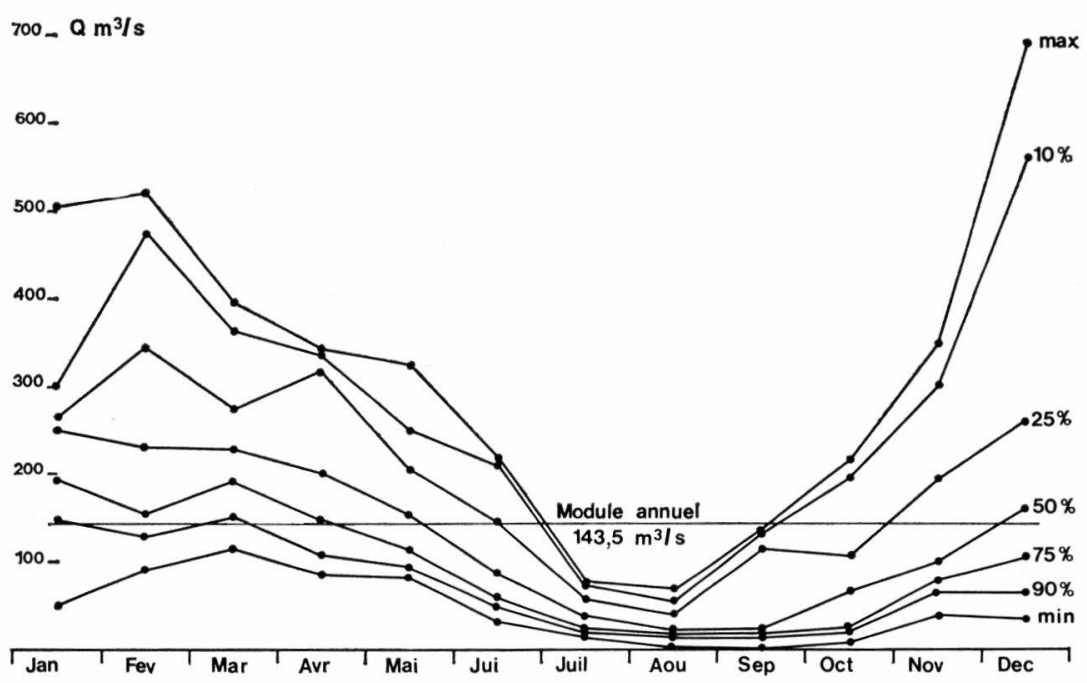

Fig. 2. - Courbes des débits mensuels à Cahors d'après leur fréquence (période 1962-1975).

L'écoulement naturel du Lot correspond à $46 \%$ des précipitations tombées sur le bassin (soit $986 \mathrm{~mm}$ en moyenne annuelle pour la période de 1971 à 1975). Ce pourcentage est voisin de ceux atteints pour la Garonne à Bordeaux - $41 \%$ - et pour l'Ebre à Tortosa $43 \%$ - (Davy 1977) ; mais il varie le long de la rivière et selon les saisons (Tableau I).

TABLEAU I. - Coefficients d'écoulement (pourcentage par rapport aux précipitations tombées sur le bassin) du Lot en régime naturel (période 1971-1975).

\begin{tabular}{lccccc}
\hline & Truyère & Haut Lot & $\begin{array}{c}\text { d'Entraygues } \\
\text { à Livinhac }\end{array}$ & $\begin{array}{c}\text { de Livinhac } \\
\text { à Cahors }\end{array}$ & $\begin{array}{c}\text { Ensemble } \\
\text { du bassin } \\
\text { en amont } \\
\text { de Cahors }\end{array}$ \\
\hline Hiver & 86 & 73 & 55 & 49 & 68 \\
Printemps & 58 & 56 & 44 & 34 & 49 \\
Eté & 18 & 16 & 12 & 13 & 15 \\
Automne & 62 & 47 & 29 & 31 & 46 \\
Total année & 56 & 50 & 36 & 32 & 46 \\
\hline
\end{tabular}

Le régime du Lot moyen et inférieur dépend étroitement du bassin supérieur (fig. 3). C'est ainsi que la partie du bassin située en amont d'Entraygues, soit $46 \%$ de la superficie totale du bassin versant, assure les $2 / 3$ du débit du Lot à Villeneuve (Tableau II). La Truyère contribue en moyenne pour $63 \%$ au débit annuel du Lot à Entraygues. Son bassin, à substratum peu perméable, présente des coefficients d'écoulement en toute saison légèrement supérieurs à ceux du 

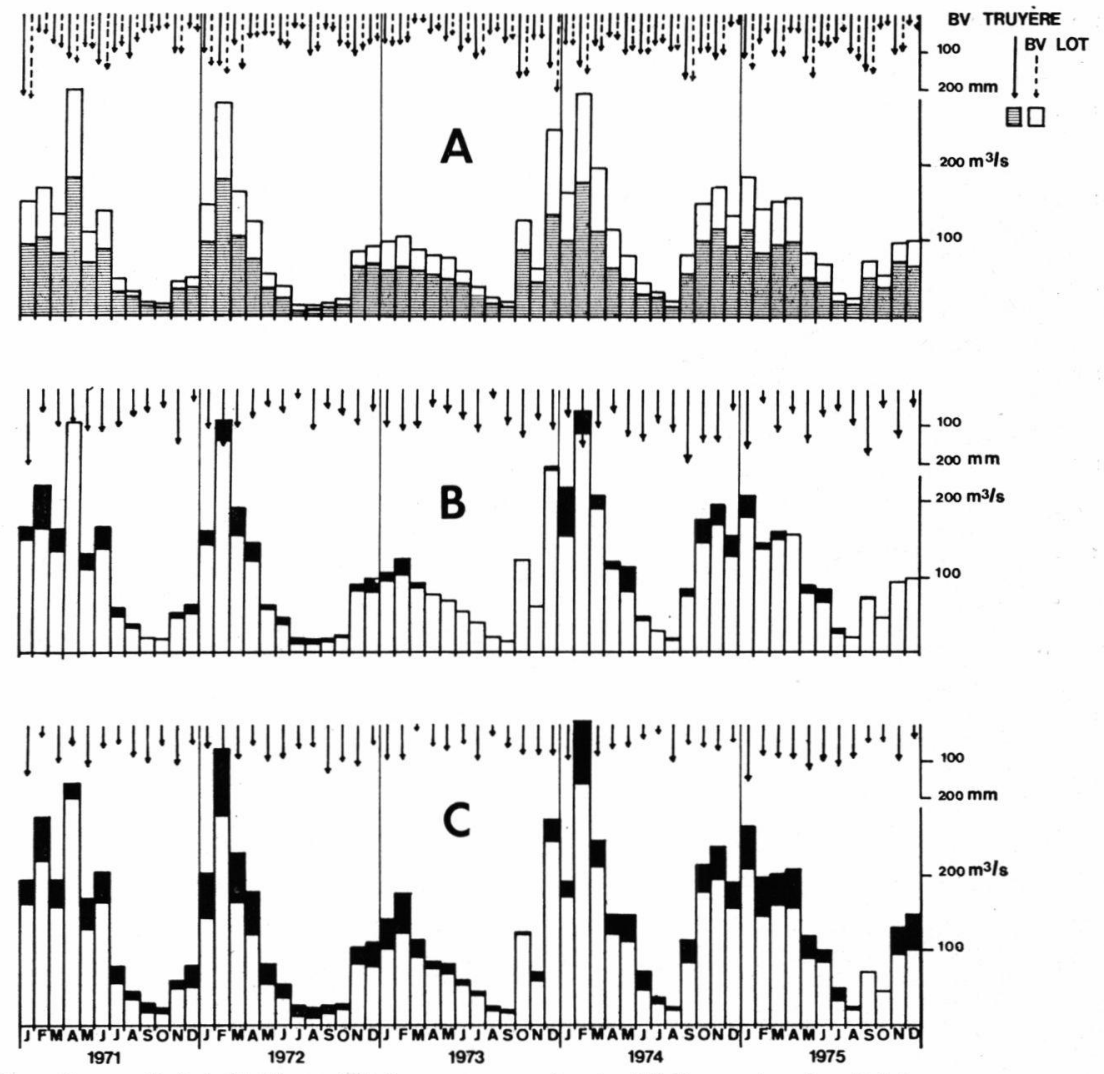

Fig. 3. - Précipitation (flèches en $\mathrm{mm}$ ) et débits naturels (histogrammes, en $\left.\mathrm{m}^{3} / \mathrm{s}\right)$ du Lot. A : à Entraygues $(\mathrm{km} \mathrm{178);} \mathrm{B} \mathrm{:} \mathrm{à} \mathrm{Livinhac}(\mathrm{km} \mathrm{216);C} \mathrm{:} \mathrm{à} \mathrm{Cahors}$ $(\mathrm{km} \mathrm{330)}$. En noir: débits apportés entre Entraygues et Livinhac (B), entre Livinhac et Cahors (C).

bassin du Haut Lot (Tableau I). Les apports des affluents en aval d'Entraygues sont faibles : le Célé atteint 10 à $14 \%$ du débit du Lot, le Dourdou 3 à $7 \%$, le Riou Mort et le Vert $1 \%$. Au total, les apports spécifiques sont de l'ordre de $10 \mathrm{l} / \mathrm{s} / \mathrm{km}^{2}$ entre Entraygues et Villeneuve contre $17,8 \mathrm{l} / \mathrm{s} / \mathrm{km}^{2}$ pour le bassin supérieur.

Tableau II. - Relations entre le débit du Lot à Entraygues (km 178) (X) et le débit en quatre stations aval $(\mathrm{Y})$.

\begin{tabular}{lll}
\hline Livinhac $(\mathrm{km} \mathrm{216)}$ & $\mathrm{Y}=1,16 \mathrm{X}-1,5$ & $\mathrm{r}=0,99$ \\
Cajarc $(\mathrm{km} \mathrm{271)}$ & $\mathrm{Y}=1,17 \mathrm{X}-5,5$ & $\mathrm{r}=0.99$ \\
Cahors $(\mathrm{km} \mathrm{330)}$ & $\mathrm{Y}=1,41 \mathrm{X}-1,1$ & $\mathrm{r}=0,98$ \\
Villeneuve $(\mathrm{km} \mathrm{433)}$ & $\mathrm{Y}=1,63 \mathrm{X}-0,8$ & $\mathrm{r}=0,97$ \\
\hline
\end{tabular}


Des mesures supplémentaires, faites entre Livinhac et Cajarc, n'ont pas permis de déceler les pertes de $5 \mathrm{~m}^{3} / \mathrm{s}$ environ paraissant exister entre les deux stations (Tableau II). Cette anomalie, attribuée en première hypothèse à la présence de massifs karstiques, pourrait résulter d'un manque de sensibilité de la station de Cajarc.

Les débits naturels $(\mathrm{Qn})$ ont été comparés aux précipitations utiles $(\mathrm{Pu})$ afin de déterminer le comportement hydrologique des diverses portions du bassin versant. Pour chaque mois, Pu correspond à la différence : précipitation $(\mathrm{P})$ - évaporation potentielle (Ep). Ep peut être évalué par la formule de Thornthwaite (1948) à partir des températures moyennes mensuelles. L'excès de précipitations sur l'évapotranspiration $(\mathrm{Pu}>\mathrm{o})$ participe à l'écoulement et à la reconstitution des réserves hydriques des sols après une période de déficit; les valeurs négatives du $\mathrm{Pu}$ indiquent que l'évapotranspiration porte sur la quantité d'eau précipitée au cours du mois et sur la réserve d'eau des sols.

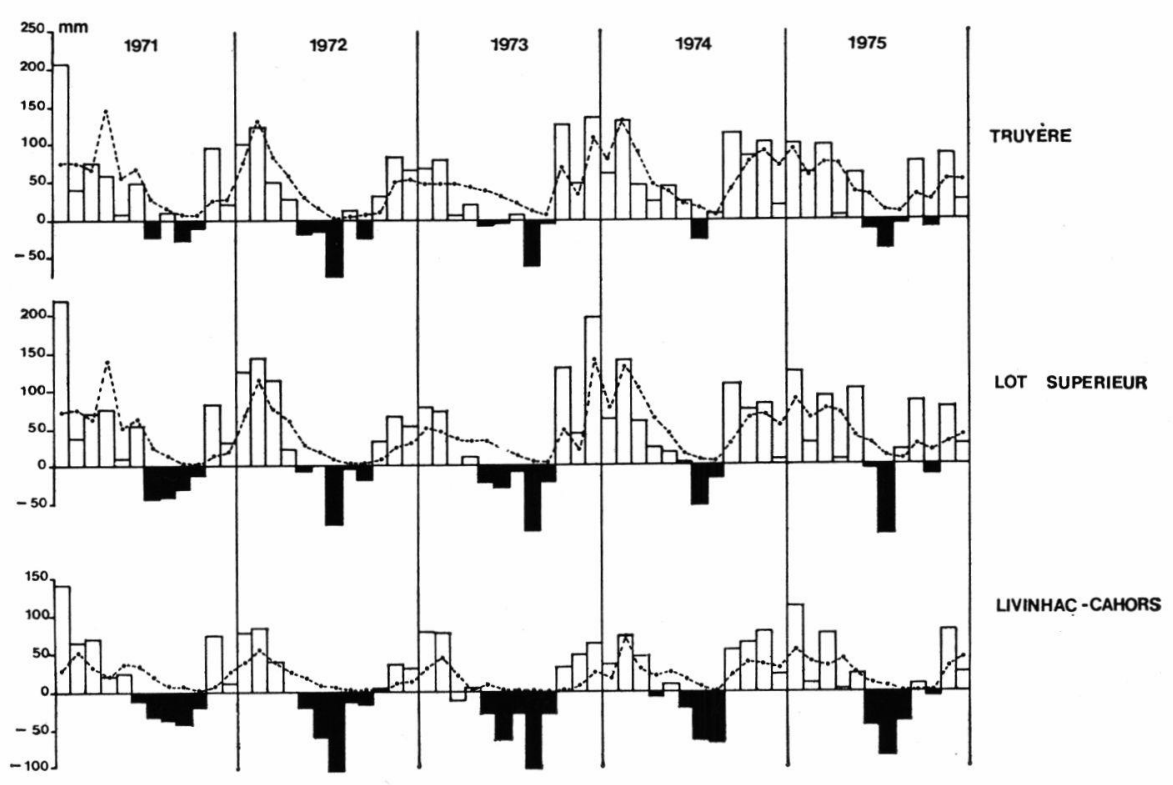

Fig. 4. - Débits naturels mensuels (courbe en tirets) et précipitations utiles (histogrammes; en noir : déficits) dans les bassins de la Truyère, du Haut Lot (ou Lot supérieur) et du Lot moyen entre Livinhac et Cahors.

L'examen des courbes de la figure 4 montre que le comportement hydrologique du bassin de la Truyère diffère peu de celui du Lot supérieur (ou Haut Lot) ; dans ce dernier, l'influence méditerranéenne se traduit cependant par une légère augmentation du déficit estival. Au contraire, la partie aval du bassin est beaucoup plus largement déficitaire : le volume des précipitations utiles y est moins élevé en hiver ; 
en été, l'évapotranspiration épuise les réserves hydriques des sols et diminue d'autant plus les débits d'étiages que l'hiver a été moins pluvieux.

\section{LES DEBITS INFLUENCES}

Les débits sont influencés par les grands barrages EDF construits de 1933 à 1962 sur le Haut Lot et la Truyère. Les retenues correspondantes ont une capacité de stockage de $22.10^{6} \mathrm{~m}^{3}$ sur le Haut Lot (Castelnau et Golinhac) et de $538.10^{\circ}$ sur la Truyère (Grandval, Sarrans, Brommat, Lardy, Maury, Couesque, Cambeyrac).

$\mathrm{Au}$ cours de l'été existe une tendance générale au déstockage sur la Truyère, tandis que les réserves relativement faibles du Haut Lot sont maintenues (Tableau III). Le plus souvent, les déstockages des

TABLEAU III. - Variation des stocks (en $\mathrm{hm}^{3}$ ) dans les barrages de la Truyère et du Lot au cours de l'été.

\begin{tabular}{cccc}
\hline Année & Truyère & Lot & Total \\
\hline 1962 & $-18,8$ & $-31,2$ & $-50,0$ \\
1963 & $+59,0$ & $+11,7$ & $+70,7$ \\
1964 & $-10,7$ & +10.4 & $\pm 0,3$ \\
1965 & $-92,2$ & $+3,6$ & $-88,6$ \\
1966 & $-2,5$ & $+4,7$ & $\pm 9,2$ \\
1967 & $-97,2$ & $+5,3$ & $+17,9$ \\
1968 & $+6,2$ & $+11,7$ & $-43,9$ \\
1969 & $-49,1$ & $+5,2$ & $-104,6$ \\
1970 & $-90,4$ & $-14,2$ & $-70,4$ \\
1971 & $-96,1$ & $+5,7$ & $+53,22$ \\
1972 & $-64,7$ & $-7,1$ & $+60,2$ \\
1973 & $+53,3$ & $-0,08$ & $-20,4$ \\
1974 & $+55,3$ & $+4,9$ & $-25,55$ \\
1975 & $-26,7$ & $+6,3$ & \\
Moyenne & $-26,7$ & $+1,21$ & \\
\hline
\end{tabular}

barrages du Haut Lot et de la Truyère ne sont donc pas synchronisés ; ceci influe sur la qualité chimique de l'eau. Par ailleurs, la gestion des stocks varie d'une année à l'autre, sans rapport avec l'importance de l'étiage ou des précipitations hivernales. Ainsi, selon les années, les débits naturels du Lot en été sont amputés ou accrus d'un volume d'eau variant entre 0 et 100 millions de $\mathrm{m}^{3}$.

Les grands barrages ont tendance à emmagasiner l'eau à la fin de l'hiver. Ils doivent en principe assurer la production d'électricité aux heures de pointe et, pour cela, ne débitent qu'à certaines heures de la journée. Leur exploitation modifie donc les débits à l'aval d'Entraygues, notamment en période d'étiage. Sur une base mensuelle, les débits d'étiage influencés (réels) sont réduits une année sur deux de 
5 à $46 \%$ (25\% en moyenne) par rapport aux débits naturels (Tableau IV). Dans $50 \%$ des cas, au contraire, les débits influencés sont augmentés de 17 à $103 \%$ ( $60 \%$ en moyenne) par rapport aux débits naturels. Sur une période de 14 ans, ce renforcement des débits d'étiage correspond en moyenne à $+8 \%$ des débits naturels. La situation diffère nettement si l'on se réfère à des périodes plus courtes : pour les débits d'étiage influencés calculés sur une période de 5 jours, l'influence des barrages aggrave les débits d'étiage dans plus de 9 années sur 10 ( $-36 \%$ par rapport aux débits naturels).

Tableau IV. - Comparaison des débits d'étiage mensuels en régime naturel et en régime influencé : station d'Entraygues.

\begin{tabular}{cccc}
\hline Année & $\begin{array}{c}\text { Etiage naturel } \\
\mathrm{m}^{3} / \mathrm{s}\end{array}$ & $\begin{array}{c}\text { Etiage influencé } \\
\mathrm{m}^{3} / \mathrm{s}\end{array}$ & $\begin{array}{c}\mathrm{A} \\
\mathrm{m}^{3} / \mathrm{s}\end{array}$ \\
\hline 1962 & 4,5 & $\mathbf{7 , 6}$ & +3.1 \\
1963 & 43,6 & 34,2 & $-9,4$ \\
1964 & 10,3 & $\mathbf{7 , 1}$ & $-3,1$ \\
1965 & 15,1 & $\mathbf{3 0 , 1}$ & $+15,0$ \\
1966 & 9,8 & $\mathbf{1 1 , 5}$ & $+1,7$ \\
1967 & 13,1 & $\mathbf{7 , 1}$ & $-\mathbf{6 , 0}$ \\
1968 & 24,1 & $\mathbf{1 8 , 7}$ & $-5,4$ \\
1969 & 27,0 & $\mathbf{3 3 , 5}$ & $+6,5$ \\
1970 & 9,7 & $\mathbf{1 7 , 7}$ & $+8,0$ \\
1971 & 15,5 & $\mathbf{3 1 , 4}$ & $+15,9$ \\
1972 & 11,2 & $\mathbf{1 4 , 2}$ & $+3,0$ \\
1973 & 16,35 & 15,5 & $-0,85$ \\
1974 & 17,55 & 12,95 & $-4,6$ \\
1975 & 20,25 & $\mathbf{1 5 , 2}$ & $-5,05$ \\
Moyenne & 17,0 & 18,34 & $+1,34$ \\
\hline
\end{tabular}

A l'étiage, les barrages relâchent un débit de base égal à $6 \mathrm{~m}^{3} / \mathrm{s}$ à Entraygues. Sur ce débit de base se superposent pendant 4 à 6 heures par jour des pointes de débit provenant soit du Haut Lot $\left(40 \mathrm{~m}^{3} / \mathrm{s}\right)$ soit de la Truyère $\left(60 \mathrm{~m}^{3} / \mathrm{s}\right)$. Par suite, les débits instantanés subissent d'importantes fluctuations sur les cours moyen et inférieur. Par ailleurs, ces pulsations tendent à renforcer les débits journaliers moyens.

En aval d'Entraygues, les débits sont remodulés de façon anarchique par 17 petites usines installées sur des seuils. Réglementairement, la plupart de ces usines devraient fonctionner au fil de l'eau, c'est-àdire sans modifier la vitesse ou le débit instantané de la rivière. En fait, au moment de l'étiage, elles fonctionnent par marnages de 20 à $50 \mathrm{~cm}$ en moyenne. Ceci se traduit par de brusques fluctuations de débit qui, par leur importance, couvrent largement les variations occasionnées par les lâchures des grands barrages de l'amont. 


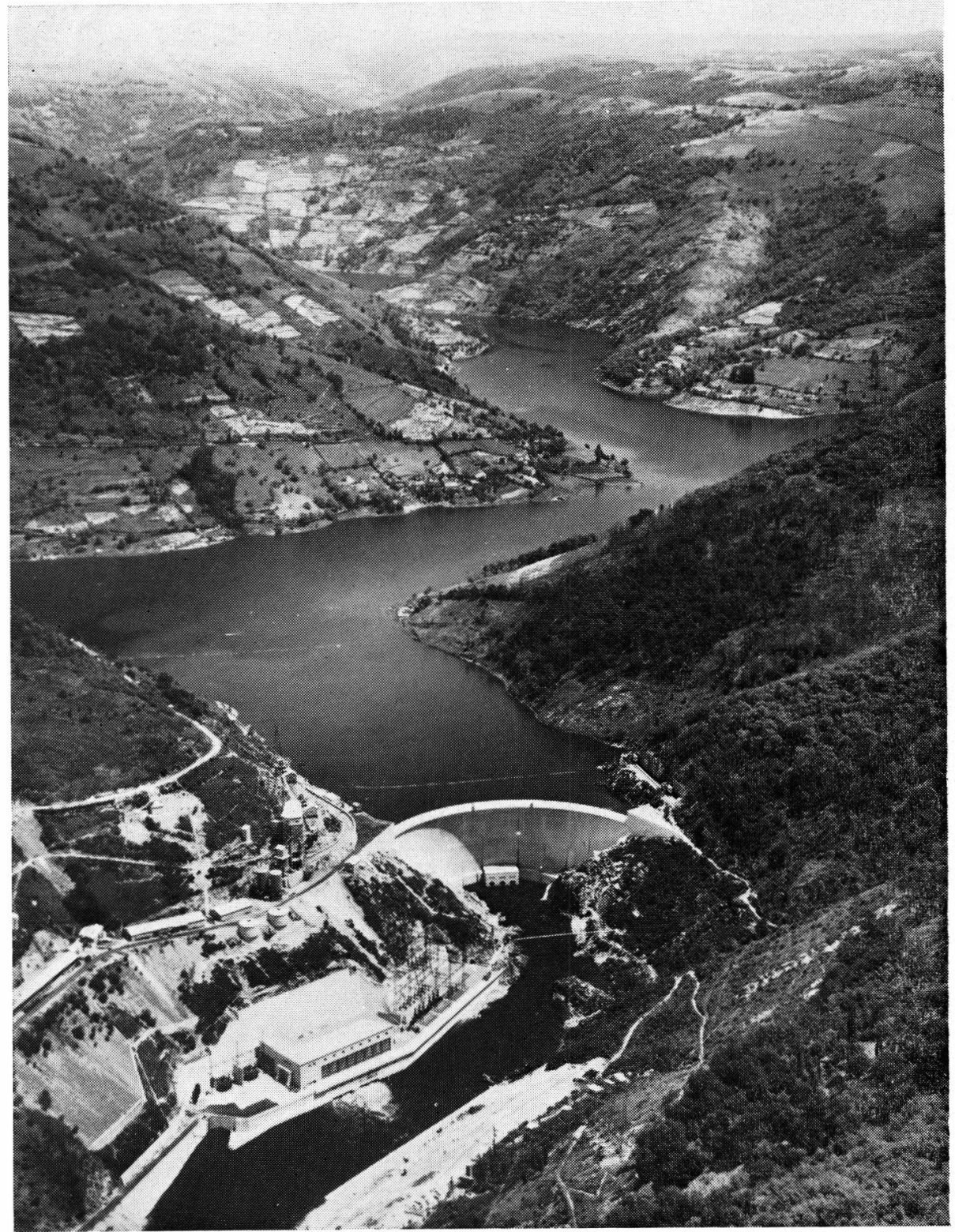

Réservoir de Couesque (20 millions de $\mathrm{m}^{3}$ ) sur la Truyère. (Photo R. Delvert). 

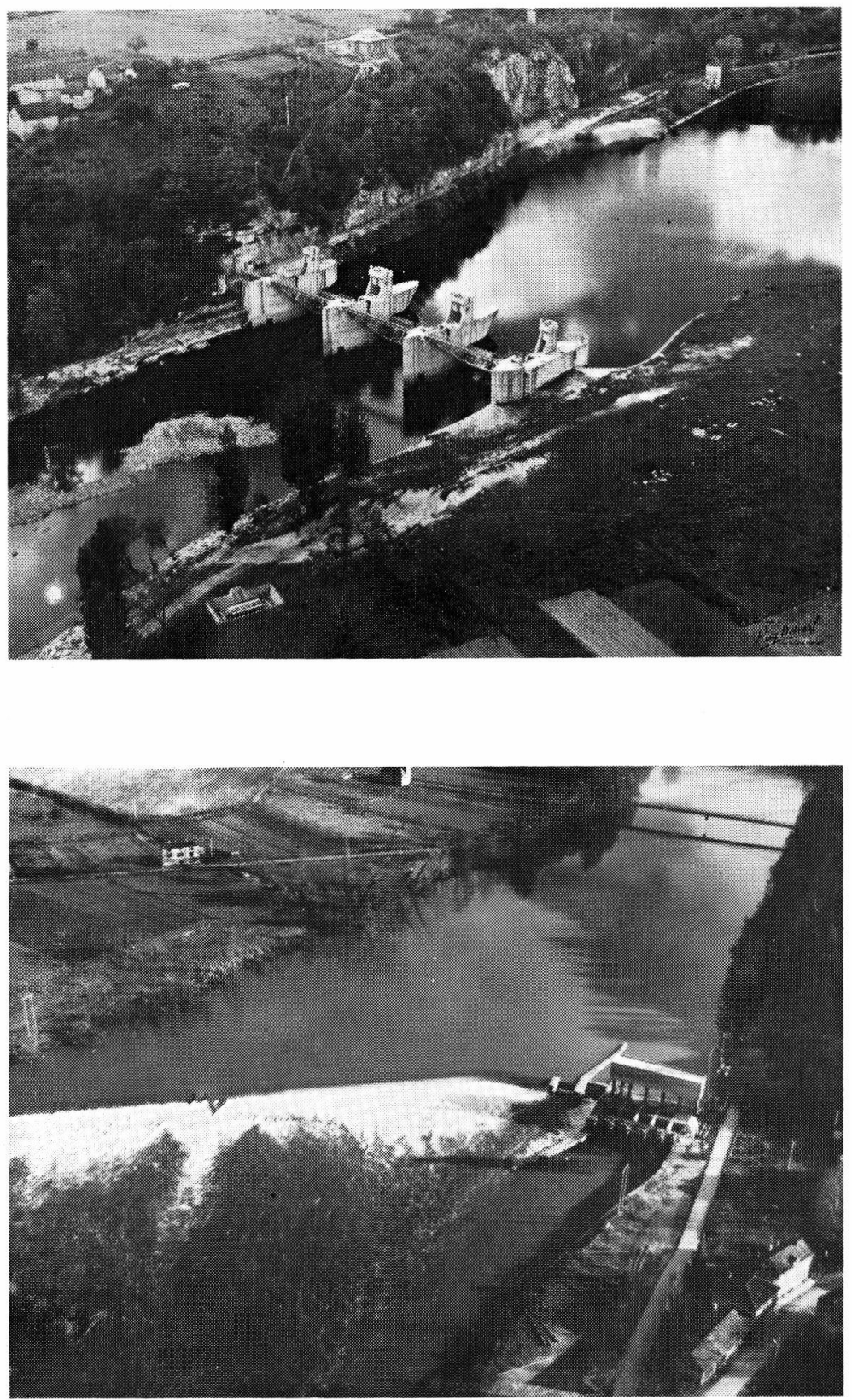

Exemples d'ouvrages sur le Lot: barrage de Cajarc (en haut) et chaussée d'Albas (en bas). (Photos R. Delvert). 
La zone canalisée du Lot est donc assimilable à une succession de biefs séparés par des seuils. Pour l'ensemble de cette zone, un modèle mathématique des écoulements (Sogreah 1975) permet de déterminer, bief par bief, pour un débit donné :

- la vitesse moyenne et le temps de renouvellement de l'eau lié à cette vitesse,

- le temps de propagation des ondes de débit.

250 - $\mathrm{Km}$ depuis ENTRAYGUES

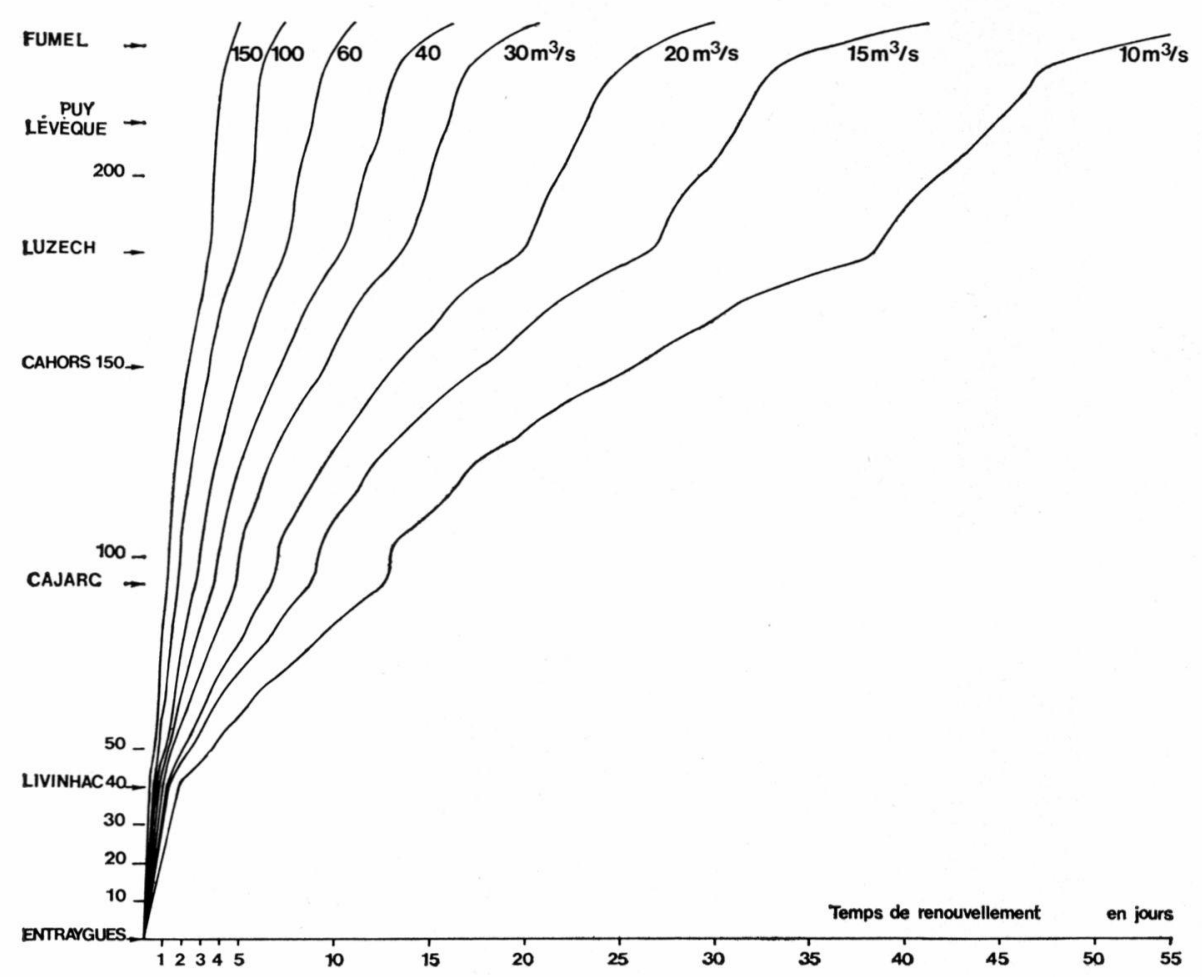

FIG. 5. - Evolution des temps de parcours de la masse d'eau (temps de renouvellement cumulés) entre Entraygues et Fumel pour différents débits.

La figure 5 indique l'évolution des temps de parcours de la masse d'eau (c'est-à-dire les temps de renouvellement cumulés) d'Entraygues à Fumel pour différentes valeurs du débit. En chaque point, la vitesse moyenne de déplacement de la masse d'eau augmente comme une fonction puissance du débit. Quant aux ondes de débit, elles se propagent beaucoup plus rapidement de bief en bief.

Divers prélèvements aggravent la sévérité des étiages du Lot. Ils correspondent aux besoins domestiques et industriels ainsi qu'aux besoins en eau d'irrigation; ils sont localisés essentiellement dans la 
moyenne et dans la basse vallée. Un volume pouvant atteindre $6 \mathrm{~m}^{3} / \mathrm{s}$ est ainsi prélevé pendant l'été.

\section{CONCLUSION}

La situation géographique du bassin versant du Lot détermine un régime hydrologique dont les caractéristiques (hautes eaux et crues importantes en saison froide, sévérité des étiages estivaux) s'apparentent à celles des rivières de type méditerranéen. L'essentiel de l'alimentation du Lot est assuré par les précipitations qui tombent dans la partie montagneuse du bassin.

Le Lot apparaît ainsi comme une rivière à régime très irrégulier et cette irrégularité n'est que très partiellement atténuée par le fonctionnement des grands ouvrages hydroélectriques de l'amont et des usines qui équipent le Lot moyen. Les études réalisées par ailleurs montrent que ces caractéristiques hydrologiques commandent en grande partie la qualité des eaux de la rivière.

\section{TRAVAUX CITES}

Davy (L.). 1977. - L'Ebre: étude hydrologique (résumé). Houille blanche, 1 : 59-66.

S.O.G.R.E.A.H. 1975. - Etiages du Lot moyen. Etude sur modèle mathématique de l'exploitation des usines. Document $n^{\circ} 11792$ (non publié). Grenoble.

ThoRnthWAITE (C. W.). 1948. - An approach toward a rational classification of climate. Geograph. Rev. $33:$ 55-94. 